\title{
Modelling particle movement in conical guide of seeder pneumatic pipe
}

\author{
Vladimir Zaitsev ${ }^{1}$, Artem Kravtsov ${ }^{1, *}$, and Vladimir Konovalovi ${ }^{1}$ \\ ${ }^{1}$ Penza State Technological University, 1A/11t, Baydukova / Gagarina str., 440039, Penza, Russia
}

\begin{abstract}
In the course of the study, methods for ensuring the centering of particles of bulk material in the air flow moving in the pneumatic duct of the seeder were investigated. To solve this problem, it is proposed to use a conical confusor. The aim of the study was to obtain the functional dependences of the movement of particles in a conical airflow guide (confusor) for the conditions of transportation of the sown particles on the basis of force analysis and to identify the nature of the movement of the sown particles in a tapering air flow. During the study, to describe the motion of particles in a vertical tapering pipe, a system of expressions was substantiated. The developed mathematical model of particle motion in a conical air flow, implemented in the MathCAD mathematical package, allows calculating both the particle trajectory and the velocity parameters of the air flow and the particles to be sown. The digital calculation results in the MathCAD program are in good agreement with the finite element calculations. The magnitude of the error in air velocity is less than $1 \%$. The differences in the velocities of the transported particles in the calculation options do not exceed 7\%. The installation of a conical guide helps to focus the flow of particles in the central part of the narrowed air line. In this case, part of the particles in the central part of the guide will retain the initial longitudinal trajectory. The angle at the apex of the cone and the parameters of the particles affect the speed and angle of the tangent contact of the particle with the guide.
\end{abstract}

\section{Introduction}

Pneumatic transport is widely used in various sectors of economy [1]. Its theoretical justification is quite well known [1,2]. Due to the specifics of the tasks, the pneumatic system of seeders has a number of features of their work, for example, the flow rate preventing the destruction of seeds [3], centered supply of material to the distribution head [4] ensuring a uniform flow of material along bends [5], minimal hydraulic losses [6] ensuring the economical functioning of the seeder. Various aspects of grain movement in the pneumatic pipe of a seeder are considered in the works of a large number of authors.

In their work, Hilton et al. [7] present a description of the process of transporting bulk material in a pneumatic system. In [2], the theoretical foundations of modelling in the Comsol Multiphysics environment are described, as well as mathematical equations describing the

\footnotetext{
*Corresponding author: artem-kravtsov-penzgtu@yandex.ru
} 
laws of motion of the air flow and material particles in it. In [8], the results of modelling a seeder pneumatic system in the OpenFOAM package were considered.

Basing on the theoretical [2;8;9] and experimental [3] results of the study of seeders' pneumatic systems, the presence of a displacement of the flow relative to the axis of the vertical pipe and distributor due to the presence of the installed supply elbow was ascertained.

To increase the uniformity of the distribution of particles over the cross cut of the pipeline, various structural elements are used, including turbulators $[4 ; 10]$. These elements show their effectiveness to some extent. However, they have a fairly low manufacturability. Moreover, the centering of the flow relative to the axis of the pipeline is achieved by dispersing the moving material in the internal volume of the pipe. This imposes certain requirements related to the geometric parameters of the pipe, the velocity of the air flow and the slope angle of the field.

An urgent task is to consider the possibility of replacing the turbulator with a more technologically advanced confuser or confuser-diffuser bundle. Their use will reduce the length of the vertical section of the pneumatic pipe. This can further increase the efficiency of the pneumatic system by focusing the flight of particles to the desired location of the pneumatic pipe to reduce the uneven distribution of particles along the bends.

The aim of the study was to obtain functional dependences of the movement of particles in a conical airflow guide (confuser) for the conditions of transporting the sown particles on the basis of force analysis and identification of the nature of the movement of the sown particles in a tapering air flow.

\section{Research methods}

The research methodology provided for the theoretical justification of the particle motion parameters in a conical tapering (conical) air flow (confuser) of the pneumatic pipe, as well as checking the obtained expressions based on the implementation of the mathematical model in the MathCAD program by comparing numerical results with modelling a similar process using the finite volume method.

To determine the velocity and the law of a particle motion along an inclined vertical wall of the pneumatic pipe, the air flow movement along the vertical conical section of the pneumatic pipe in the upward direction is considered (Figure 1).

To determine the parameters of the air flow and the parameters of a particle motion, the following assumptions are introduced: 1) to simplify the calculation, we will consider the velocity of the air flow as evenly distributed along the base of the cone; 2) the angle of inclination of the air flow stream lines at the inclined wall of the cone is equal to the angle of the cone inclination $\alpha$ and decreases in proportion to the ratio of the distances from the position of the particle to the inclined wall in cross cut relative to the axis of symmetry of the cone. The streamline is vertical on the axis of symmetry of the cone (Figure 1). The parameters of the air flow: air flow velocity at the inlet of the conical section $W_{b}, \mathrm{~m} / \mathrm{s}$; total air pressure at the inlet of the conical section of the pneumatic pipe $P_{b}, \mathrm{~Pa}$; air density at the inlet of the conical section $\rho_{a b}, \mathrm{~kg} / \mathrm{m}^{3}$; air flow velocity at the outlet of the conical section $W_{t}, \mathrm{~m} / \mathrm{s}$; total air pressure at the outlet of the conical section of the pneumatic pipe $P_{t}, \mathrm{~Pa}$; air density at the outlet of the conical section $\rho_{a t}, \mathrm{~kg} / \mathrm{m} 3$; air flow rate in the current cut of the conical section $W_{i}, \mathrm{~m} / \mathrm{s}$; total air pressure in the current cut of the conical section of the pneumatic pipe $P_{i}, \mathrm{~Pa}$; air density in the current cut of the conical section $\rho_{a i}, \mathrm{~kg} / \mathrm{m}^{3}$. 


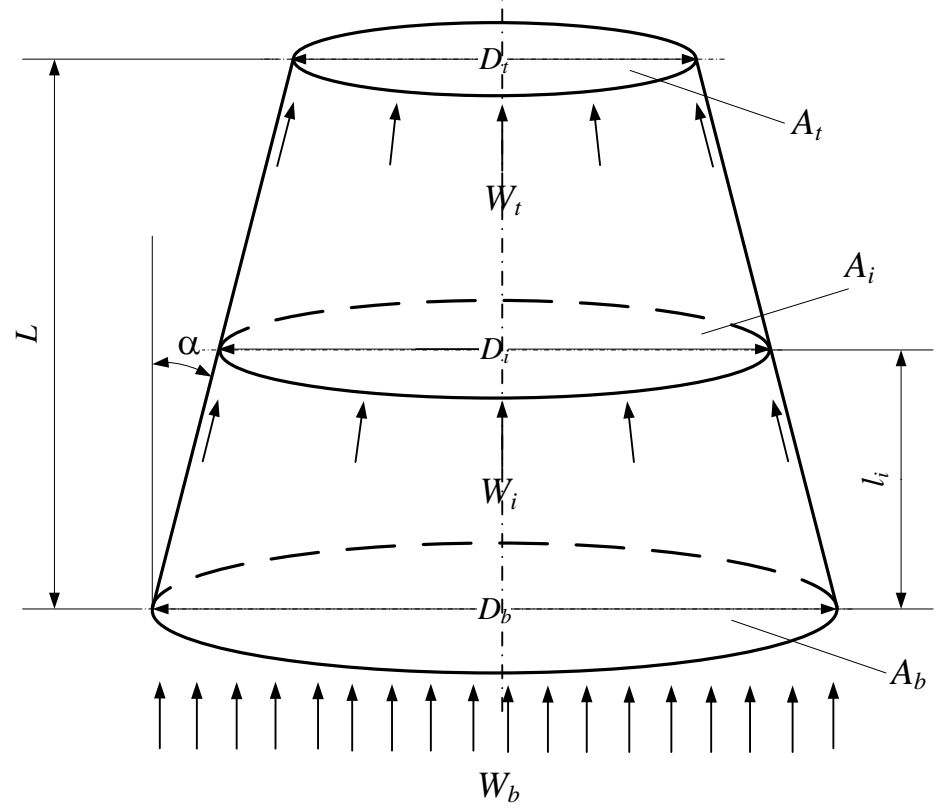

Fig. 1. Vertical conical section of the pipeline (confuser): $D_{b}$ - diameter of the base of the cone (diameter of the inlet of the conical section), m; ${ }_{b}$ - cross-cut area of the base of the cone, m2; ${ }^{D_{t}}$ diameter of the apex of the cone (diameter of the outlet of the conical section), m; $A_{t}$-cross-cut area of the apex of the cone, m2; ${ }{ }_{i}$ - diameter of the current cross cut of the cone, m; $A_{i}$-area of the current cross cut of the cone, $\mathrm{m} 2 ; L_{-}$full height of the truncated cone (the length of the conical vertical section of the pneumatic pipe), $\mathrm{m} ;{ }_{i}$ - height of the current cross cut of the truncated cone (the current length of the conical vertical section of the pneumatic pipe), $\mathrm{m} ; \alpha_{- \text {angle of inclination }}$ of the generatrix of the cone to the vertical, deg.

Based on the law of conservation of mass, the velocity of air flow in the current cut of the pneumatic pipe with a slight change in the density of the air flow is

$$
W_{i}=W_{b} \cdot \frac{A_{b}}{A_{i}},
$$

where $A_{b}=\frac{\pi \cdot D_{b}^{2}}{4}$ - area of cross cut of the cone base, $\mathrm{m} 2 ;{ }^{A_{i}=\frac{\pi \cdot D_{i}^{2}}{4}}$ - area of the current cross cut of the cone, $\mathrm{m}^{2}$.

Based on Figure 1, the diameter of the current cross cut is determined by the expression

$$
D_{i}=D_{b}-2 \cdot l_{i} \cdot \tan (\alpha) \text {. }
$$

Thus, equation (1), taking into account expression (2), will take the form

$$
W_{i}=W_{b} \cdot \frac{D_{b}^{2}}{\left(D_{b}-2 \cdot l_{i} \cdot \tan (\alpha)\right)^{2}} .
$$

Based on equations (1) and (3) one can see that with the decrease in the cross-cut area of the pneumatic pipe (with the decrease in diameter), the air flow rate increases, while with the 
increase in the velocity of the air stream, the total pressure in the cuts of the pneumatic pipe decreases.

Particle parameters: diameter $d, \mathrm{~m}$ (the second assumption is a spherical particle); particle mass $m, \mathrm{~kg}$.

Let us consider the movement of a particle along an inclined vertical wall of the pneumatic pipe (Figure 2). The particle falls on the wall of the conical vertical section of the pneumatic pipe from the vertical cylindrical section of the pneumatic pipe with the initial velocity $V_{b}=k_{l} \cdot W_{b}$, where $k_{l}=0.75-0.93$ is a coefficient of the lag of the particle from the air flow [11]. Further, the particle moves in the air flow along the wall of the conical section of the pneumatic pipe upward (partially reflecting), accelerating after braking as a result of a tangential impact against the wall.

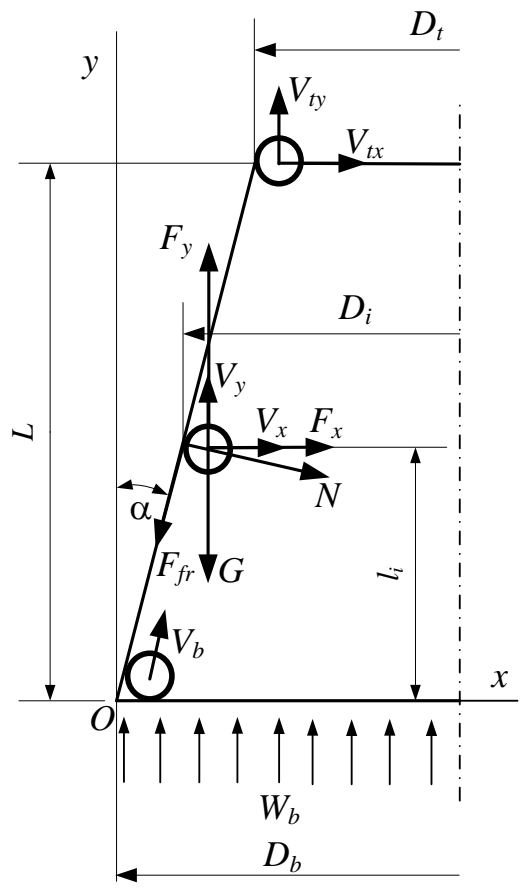

Fig. 2. The predicted scheme of particle motion along the inclined wall of the vertical section of the pneumatic pipe.

Where: $D_{b}$-diameter of the base of the cone (diameter of the inlet of the conical section), $\mathrm{m} ; D_{t}$ - diameter of the apex of the cone (diameter of the outlet of the conical section), $\mathrm{m}$; $D_{i}$ - diameter of the current cross cut of the cone, $\mathrm{m} ; A_{i}$ - area of the current cross cut of the cone, $\mathrm{m}^{2} ; L$ - full height of the truncated cone (the length of the conical vertical section of the pneumatic pipe), $\mathrm{m} ;{ }_{i}$ - height of the current cross cut of the truncated cone (the current length of the conical vertical section of the pneumatic pipe), $\mathrm{m} ; \alpha_{-}$angle of inclination of the generatrix of the cone to the vertical, deg.; $\mathrm{G}$ - gravity, N; F- aerodynamic drag force, $\mathrm{N} ; \mathrm{N}$ - normal support reaction force, $\mathrm{N} ;{ }^{F_{f r}}$ - friction force, $\mathrm{N} ; \mathrm{W}_{\mathrm{b}}$ - air velocity at the inlet to the confuser, $\mathrm{m} / \mathrm{s} ; \mathrm{V}_{\mathrm{x}}, \mathrm{V}_{\mathrm{y}}$ - projections of the absolute particle velocity vector on the $\mathrm{x}, \mathrm{y}$ coordinate axes. 
When a particle moves along a pipeline wall in an air stream, gravity $G=m \cdot g$, aerodynamic drag force $F$, normal support reaction force $N$ and friction force $F_{f r}$ act on it. Other forces (Magnus force and Archimedes force, electrostatic forces, molecular interaction force, thermophoresis force and photophoresis force, turbulent mass transfer force, Basset force) are neglected due to the smallness of the particle and the length of the conical section.

The motion of a particle in the air flow can be described using Newton's second law in the vector form:

$$
m \cdot \dot{a}=\dot{G}+\dot{F}+\dot{F}_{f r}+\dot{N}
$$

where ${ }^{\bar{a}=\frac{d \bar{V}}{d t}}$ is a vector of absolute acceleration of the particle, $\mathrm{m} / \mathrm{s} 2 ;{ }^{m=\frac{\rho_{\mathrm{T}} \cdot \pi \cdot d^{3}}{6}}$ is a particle mass, $\mathrm{kg} ; \dot{V}$ is a vector of the absolute particle velocity in the current cut of the pipeline, $\mathrm{m} / \mathrm{s} ;{ }^{t}$ is a particle motion time, $\mathrm{s} ; \rho_{\mathrm{T}}$ is a particle density, $\mathrm{kg} / \mathrm{m}^{3}$.

Gravity vector:

$$
\dot{G}=m \cdot g=\frac{\rho_{\mathrm{T}} \cdot \pi \cdot d^{3}}{6} \cdot \dot{g}
$$

Air drag force [11]:

$$
\dot{F}=\frac{1}{2} \cdot k \cdot k^{\prime} \cdot \rho_{\mathrm{B}} \cdot A \cdot\left|\dot{W}_{i}-\dot{V}\right| \cdot\left(\dot{W}_{i}-\dot{V}\right)
$$

where $A=\frac{\pi \cdot d^{2}}{4}$ is an area of the cross cut of a particle, $\mathrm{m} 2 ; k$ is a coefficient of aerodynamic drag; $k^{\prime}$ is a coefficient of a particle shape.

Coefficient of aerodynamic drag is determined by the formula $[12,13]$

$$
k=\frac{24}{\operatorname{Re}} \cdot\left(1+0.17 \cdot \operatorname{Re}^{\frac{2}{3}}\right) .
$$

Reynolds number during particle motion in the air flow is determined as [11]:

$$
\operatorname{Re}=\frac{d \cdot W_{r}}{\mu},
$$

where $\mu$ is a kinematic viscosity of air, $\mathrm{m}^{2} / \mathrm{s}$.

\section{Research result}

Let us superpose the origin of the Cartesian coordinate system Oxy with the intersection of the base of the cone with the generatrix, direct the Oy axis vertically upward, the Ox axis horizontally to the right, to the longitudinal axis of the pipeline (Figure 2). Thus, the particle position at the conical vertical section of the pipeline will be determined by the $\mathrm{x}$ and $\mathrm{y}$ coordinates. Let us designate the projections of the flow velocity vector $\dot{W}_{i}$ on the $\mathrm{x}, \mathrm{y}$ coordinate axes as $\mathrm{W}_{\mathrm{x}}, \mathrm{W}_{\mathrm{y}}$, respectively, and the projections of the absolute particle velocity vector $\dot{V}$ on the $\mathrm{x}, \mathrm{y}$ coordinate axes as $\mathrm{V}_{\mathrm{x}}, \mathrm{V}_{\mathrm{y}}$, respectively. 
The projections of absolute velocity on the $\mathrm{x}$ and $\mathrm{y}$ coordinate axes are determined by the system of equations:

$$
\left\{\begin{array}{l}
V_{x}=\frac{d x}{d t} \\
V_{y}=\frac{d y}{d t}
\end{array}\right.
$$

The projections of the absolute acceleration of the particle on the Oxy axes will be written as:

$$
\left\{\begin{array}{l}
a_{x}=\frac{d V_{x}}{d t} \\
a_{y}=\frac{d V_{y}}{d t}
\end{array}\right.
$$

The modulus of the difference of the vectors $\dot{W}_{i}$ and $\vec{V}$ in expression (6), taking into account the agreed designations, will be determined as:

$$
\left|\dot{W}_{i}-\dot{V}\right|=W_{r}=\sqrt{\left(W_{y}-V_{y}\right)^{2}+\left(W_{x}-V_{x}\right)^{2}} .
$$

Given the particle cross-cut area and equations (7), (8) and (11), equation (6) will take the form

$$
\dot{F}=3 \cdot k^{\prime} \cdot \rho_{\mathrm{B}} \cdot \pi \cdot d \cdot \mu \cdot\left(1+0.17 \cdot\left(\frac{d \cdot W_{r}}{\mu}\right)^{\frac{2}{3}}\right) \cdot\left(\dot{W}_{i}-\dot{V}\right)
$$

Having projected equation (12) on the $\mathrm{x}$ and y coordinate axes, we will obtain

$$
\left\{\begin{array}{l}
F_{x}=3 \cdot k^{\prime} \cdot \rho_{\mathrm{B}} \cdot \pi \cdot d \cdot \mu \cdot\left(1+0.17 \cdot\left(\frac{d \cdot W_{r}}{\mu}\right)^{\frac{2}{3}}\right) \cdot\left(W_{x}-V_{x}\right), \\
F_{y}=3 \cdot k^{\prime} \cdot \rho_{\mathrm{B}} \cdot \pi \cdot d \cdot \mu \cdot\left(1+0.17 \cdot\left(\frac{d \cdot W_{r}}{\mu}\right)^{\frac{2}{3}}\right) \cdot\left(W_{y}-V_{y}\right) .
\end{array}\right.
$$

Having projected equation (4) on Oxy coordinate axes, we will obtain:

$$
\left\{\begin{array}{l}
m \cdot \frac{d V_{x}}{d t}=N \cdot \cos (\alpha)+F_{x}-F_{f r} \cdot \sin (\alpha) \\
m \cdot \frac{d V_{y}}{d t}=F_{y}-G-N \cdot \sin (\alpha)-F_{f r} \cdot \cos (\alpha)
\end{array}\right.
$$

where $N=F_{y} \cdot \sin (\alpha)-F_{x} \cdot \cos (\alpha)-G \cdot \sin (\alpha) \quad F_{f r}=N \cdot v, v_{- \text {friction coefficient. }}$

Then, as a result of transformations, the equation of motion of the particle (from formula 14) will take the form: 


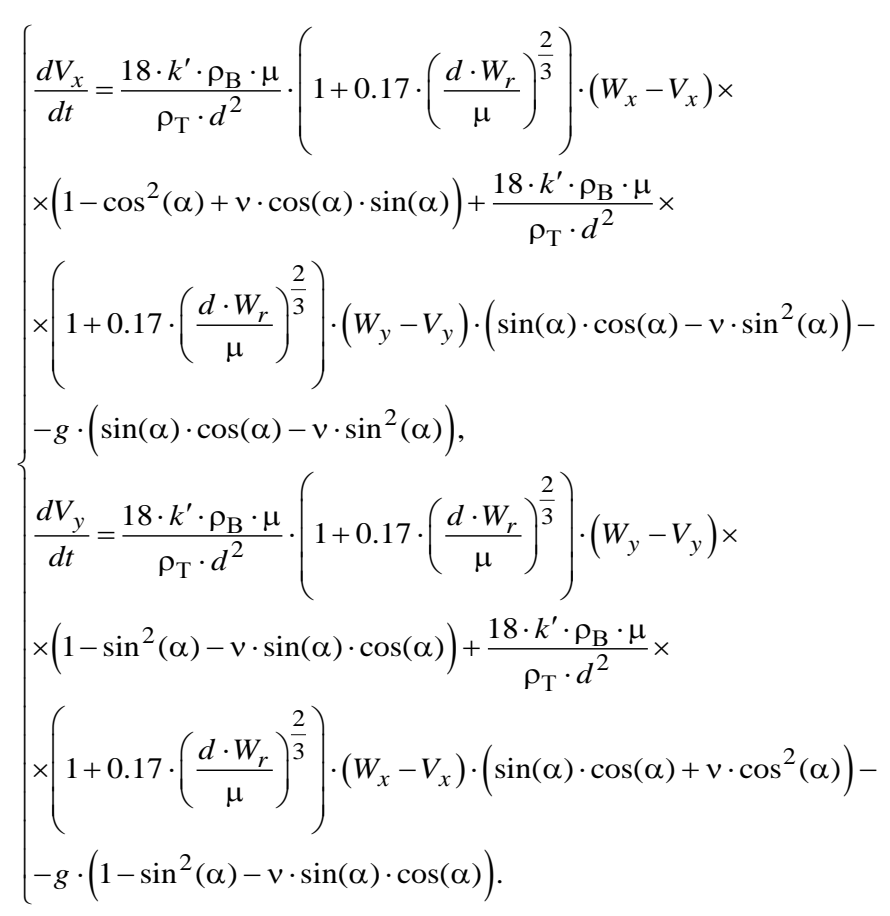

The system of equations (15), (9) and (11) describe the motion of a particle in the air flow along an inclined vertical wall of a pneumatic pipe.

The movement of a particle at a vertical conical section of a pneumatic pipe in the absence of direct contact (touch) with the wall is described by the following system of equations:

$$
\left\{\begin{array}{l}
\frac{d V_{x}}{d t}=\frac{18 \cdot k^{\prime} \cdot \rho_{\mathrm{B}} \cdot \mu}{\rho_{\mathrm{T}} \cdot d^{2}} \cdot\left(1+0.17 \cdot\left(\frac{d \cdot W_{r}}{\mu}\right)^{\frac{2}{3}}\right) \cdot\left(W_{x}-V_{x}\right), \\
\frac{d V_{y}}{d t}=\frac{18 \cdot k^{\prime} \cdot \rho_{\mathrm{B}} \cdot \mu}{\rho_{\mathrm{T}} \cdot d^{2}} \cdot\left(1+0.17 \cdot\left(\frac{d \cdot W_{r}}{\mu}\right)^{\frac{2}{3}}\right) \cdot\left(W_{y}-V_{y}\right)-g .
\end{array}\right.
$$

Numerical modelling based on the constructed mathematical expressions was carried out for the following parameters: air flow velocity at the base of the cone $W_{b}=15 \mathrm{~m} / \mathrm{s}$; air density $\rho_{\mathrm{B}}=1.29 \mathrm{~kg} / \mathrm{m}^{3}$; dynamic viscosity of air $\mu=1.5 \cdot 10^{-5} \mathrm{~Pa} \cdot \mathrm{s}$; angle of the inclination of the wall of the truncated cone $\alpha=10$ degrees; diameter of the base of the truncated cone $D_{b}=0.104 \mathrm{~m}$; truncated cone height $L=0.0865 \mathrm{~m}$; coefficient of a particle lag from the air flow $k_{l}=0.8$; particle shape coefficient $k^{\prime}=1$; coefficient of friction of a particle against a pipeline wall $v=0.1$. The results of mathematical modelling in the MathCAD program are shown in Figures 3, 4. 


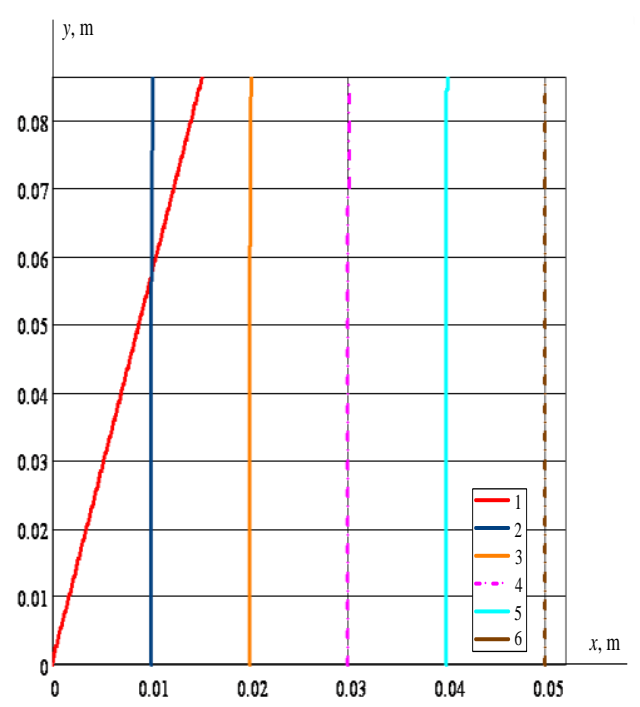

(a)

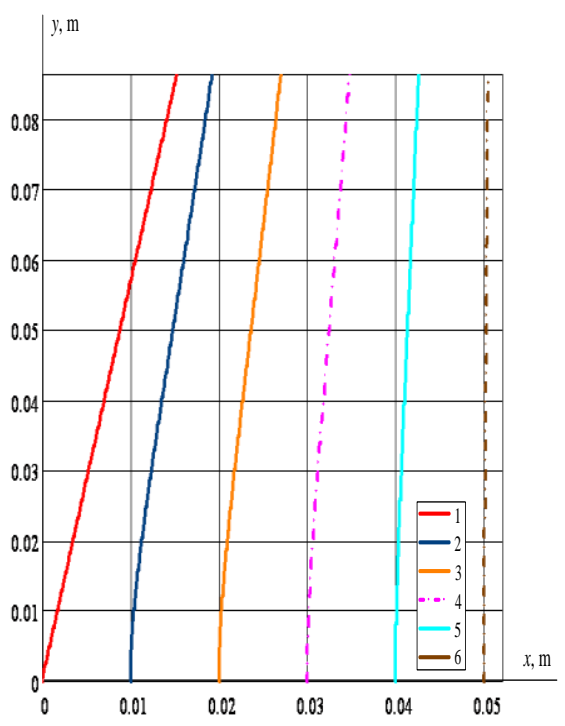

(b)

Fig. 3. Particle trajectories at a vertical conical section of a pneumatic pipe.

Where: (a) - with the particle diameter of $d=0.004 \mathrm{~m}$; particle density of $\rho_{\mathrm{T}}=1340 \mathrm{~kg} / \mathrm{m} 3$; (b) - with the particle diameter of $d=0.0001 \mathrm{~m}$; particle density of $\rho_{\mathrm{T}}=1075 \mathrm{~kg} / \mathrm{m} 3 ; 1-$ trajectory of the particle movement along an inclined conical wall of the pipeline; $2,3,4,5$, 6 - trajectories of the movement of free particles at a vertical conical section of the pipeline for different values of the initial coordinates of particles entering the conical section along the $\mathrm{x}$ axis, where the origin of the $\mathrm{x}$ axis is a wall of the initial cylindrical section, of the $\mathrm{y}$ axis - the beginning of the conical section, $\mathrm{m}$; the end of the interval for $\mathrm{x}$ is a radial axis of the pipeline, for $\mathrm{y}$ - the upper edge of the conical section.

The trajectory of particles similar to the seeds to be sown (Figure 3.a) shows an insignificant change in the radius of the particle's position in the air flow of the conical guide. This is influenced by the small length of the guide (short exposure time) and the substantial mass of a particle (the ratio of mass and cross-cut area). As a result, particles from the overlapped area of the initial pneumatic pipe tangentially hit the wall of the guide spending kinetic energy on contact with the wall and changing the direction of the flight, and focus in the direction of the axis of the pneumatic pipe (see Figure 5). Particles of the central part of the pneumatic pipe continue the initial flight trajectory. With the decrease in a particle size and its density, particles have an opportunity to move to the central part of the pneumatic pipe (Figure 3.b) with virtually no contact with the conical guide. The ability to focus is retained.

The analysis of the ratio of the particle velocities along the axis of the pneumatic pipe (y axis) and in the radial direction (x axis) for the first material shows an almost linear dependence (Figure 4.a). With the decrease in the density of the material and particle size, the influence of the air flow increases, as a result, the nature of the mutual change in velocities tends to a parabolic form of dependencies. Due to the decrease in the cross-cut area of the pneumatic pipe, the air flow rate increases. As a result, the particle accelerates - the longitudinal velocity increases from $12 \mathrm{~m} / \mathrm{s}$ in the first case to $12.4 \mathrm{~m} / \mathrm{s}$, and in the second case - to $17 \mathrm{~m} / \mathrm{s}$. 


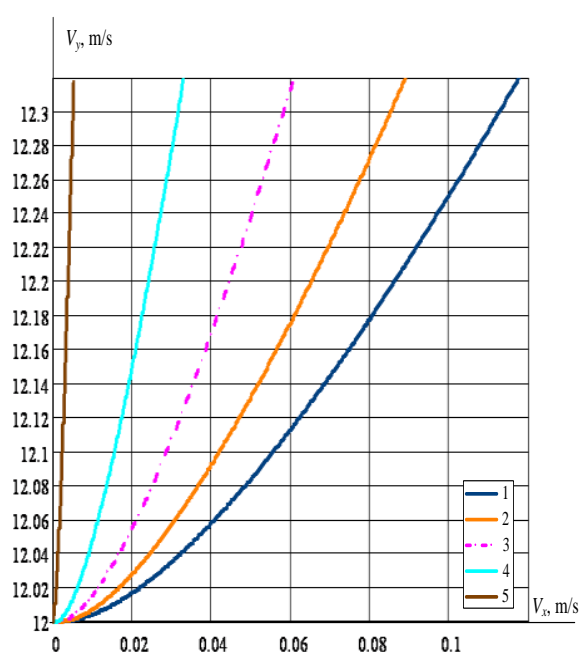

(a)

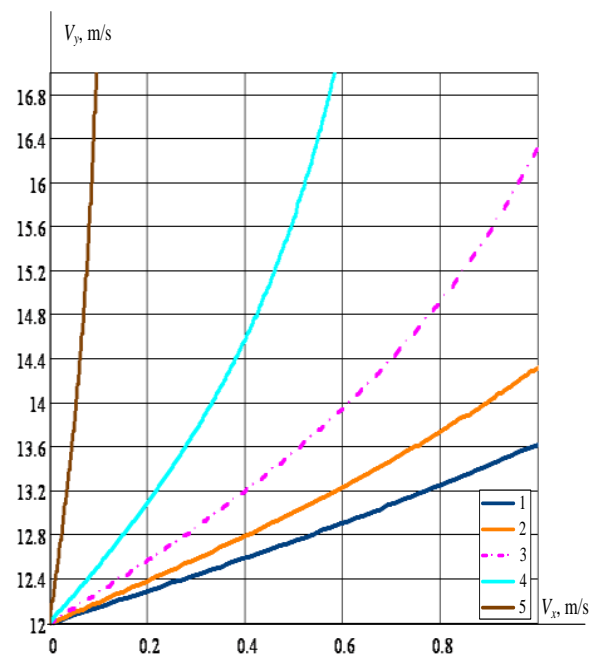

(b)

Fig. 4. Hodographs of particle velocities at a vertical conical section of a pneumatic pipe.

Where: (a) - with the particle diameter of $d=0.004 \mathrm{~m}$; particle density of $\rho_{\mathrm{T}}=1340 \mathrm{~kg} / \mathrm{m} 3$; (b) - with the particle diameter of $d=0.0001 \mathrm{~m}$; particle density of $\rho_{\mathrm{T}}=1075 \mathrm{~kg} / \mathrm{m} 3 ; 1,2,3,4,5$ - hodographs of the velocities of free particles at a vertical conical section of the pipeline for different values of the initial coordinates of entering the conical section along the $\mathrm{x}$ axis (see Figure 3).

Analysis of the modelling results (Figures 3 and 4) shows that in case of particles falling into the zone adjacent to the axis of symmetry of the cone of the pipeline, their trajectories tend to straight vertical lines (the zone is located between lines 4 and 6 in Figure 3 ) with the horizontal components of the particle velocity being insignificant (tending to zero), i.e. particles move vertically upward. Particles falling into the vertical conical section of the pipeline limited by lines 1-4 of Figure 3, when moving along the conical pipeline, gradually begin to shift from the inclined wall to the axis of the pipeline under the influence of the inclination of the air flow. In this case, the particles reach the inclined conical wall (the intersection of line 1 by the trajectories of particles 2 and 3) and, as a result of tangential collision with the wall, the velocity decreases along the y axis. The percussive effect is not considered in this paper.

The results of digital calculation in the MathCAD program (Figures 3, 4) are in good agreement with the finite element calculations (Figure 5). The magnitude of the error of the air flow velocities is less than $1 \%$. Differences in the velocities of the transported particles in the calculation options do not exceed 7\%. Partially, the differences are due, inter alia, to the difference between the uniformly distributed flow in theoretical studies and the presence of the formation of a core flow while using the finite element method, when the air velocity is lower at the walls than in the central part.

As a result of the contact, the particles (reflecting slightly from the wall) are forced to slow down and will move along the inclined wall of the conical guide (Figure 5). Due to the effect of the accelerating air flow, particles accelerate again. In Figure 5, the initial high velocity is red lines $(12 \mathrm{~m} / \mathrm{s})$, and after the contact with the wall the velocity decreases black lines $(\mathrm{m} / \mathrm{s})$. The trajectory of the flight of particles along the wall is shown by lines in a three-dimensional rather than flat image. The color background shows the air flow velocity. The average air velocity in the cross cuts of the guide varies from 15 to $30 \mathrm{~m} / \mathrm{s}$. 


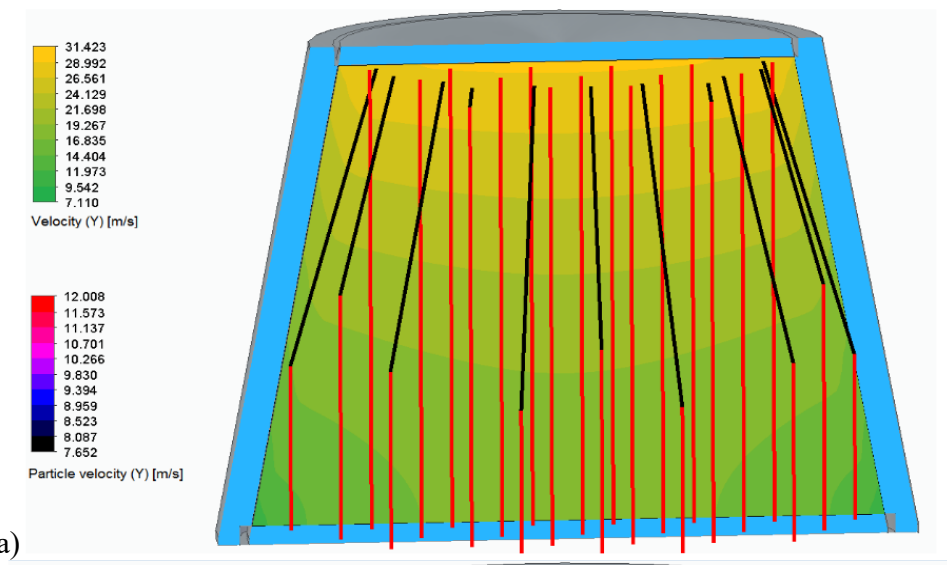

(a)

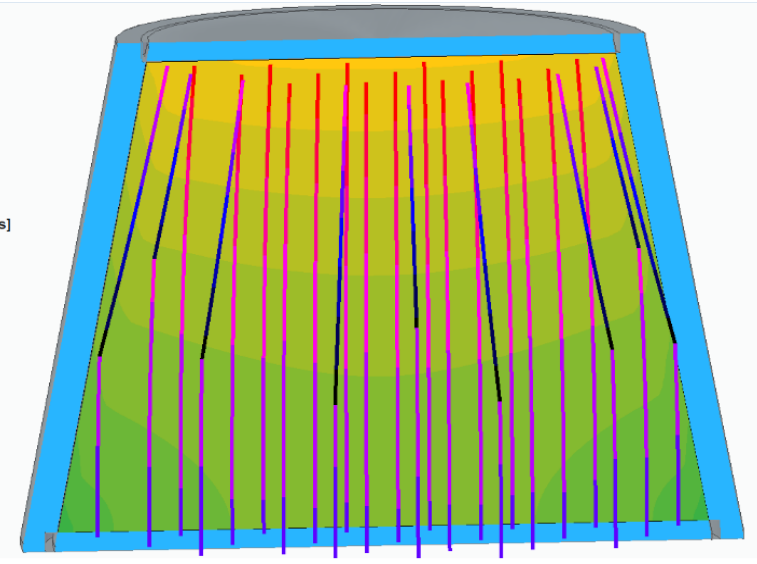

(b)
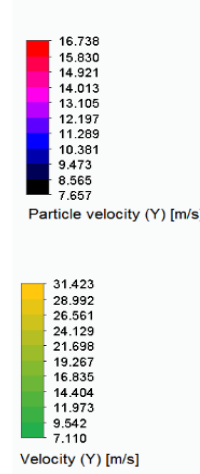

Fig. 5. The results of modelling the movement of particles in a pneumatic pipe with a conical section (confuser) using the finite volume method: (a) - with the particle diameter of $d=0.0001 \mathrm{~m}$; particle density of $\rho_{\mathrm{T}}=1340 \mathrm{~kg} / \mathrm{m}^{3}$; (b) - with the particle diameter of $d=0.0001 \mathrm{~m}$; particle density of $\rho_{\mathrm{T}}=1075 \mathrm{~kg} / \mathrm{m}^{3}$.

\section{Conclusion}

The developed mathematical model of particle motion in a conical air flow, implemented in the MathCAD mathematical package, allows calculating both the particle trajectory and the velocity parameters of the air flow and the particles being sown.

The digital results of calculation in the MathCAD program are in good agreement with the finite element calculations. The magnitude of error of the air flow velocities is less than $1 \%$. Differences in the velocities of the transported particles in calculation options do not exceed $7 \%$.

The installation of a conical guide facilitates particle flow focusing in the central part of a narrowed pneumatic pipe. A part of the particles will retain the initial longitudinal trajectory. The angle at the apex of the cone affects the velocity and angle of tangential contact of a particle with the guide. To eliminate injury of seeds, the rationale for the taper angle is subject to experimental verification. Reducing the cross-cut of the pneumatic pipe accelerates the velocity of the air flow and the velocity of particles, which requires measures to eliminate injury of seeds in the distributor of the pneumatic system along the bends. 


\section{References}

1. Yu.A. Çengel, J.M. Cimbala, Fluid Mechanics: Fundamentals and Applications (McGraw-Hill, New-York, 2014)

2. G. Bourges, M. Medina, Ist International Symposium on CFD Applications in Agriculture 1008, 2594 (2013) DOI:10.17660/ActaHortic.2013.1008.34

3. Ya.A. Lemière, Biosystems Engineering 161, $12034 \quad$ (2017) DOI:10.1016/j.biosystemseng.2017.06.015

4. X. Lei, Y. Liao, L. Wang, D. Wang, L. Yao, Q. Liao, Transactions of the Chinese Society of Agricultural Engineering 33(19), 67 (2017) DOI:10.11975/j.issn.10026819.2017.19.009

5. X. Lei, Y. Liao, Q. Zhang, L. Wang, Q. Liao, Computers and electronics in agriculture 150, 98 (2018) DOI: 10.1016/j.compag.2018.04.009

6. X. Lei, Y. Liao, W. Zhang, S. Li, D. Wang, Q. Liao, Transactions of the Chinese Society for Agricultural Machinery 48(3), 57-68 (2017) DOI:10.6041/j.issn.10001298.2017.03.007

7. J.E. Hilton, P.W. Cleary, Chemical engineering science 66(3), 231-240 (2011) DOI: 10.1016/j.ces.2010.09.034

8. M. Bayati, C. Johnston, CFD-DEM Investigation of Seed Clustering in an Air Seeder with the Immersed Boundary Method, https://www.radix-ic.com/news/radix-cfd-teamrecognized-for-work-on-seed-clustering-air-seeders/

9. A. Kravtsov, V. Konovalov, V. Zaytsev, M. Dontsova, Bulletin Samara state agricultural academy 2, 75-83 (2019) DOI:10.12737/article_5cdbc098156d56.12929222 https://elibrary.ru/item.asp?id=38247552

10. Y. Dai, X. Luo, Z. Wang, S. Zeng, Y. Zang, W. Yang, M. Zhang, B. Wang, H. Xing Nongye Gongcheng Xuebao, Transactions of the Chinese Society of Agricultural Engineering 32(24), 36-42 (2016) DOI: 10.11975/j.issn.1002-6819.2016.24.005

11. S. Mudarisov, E. Khasanov, Z. Rakhimov, I. Gabitov, I. Badretdinov, I. Farchutdinov, F. Gallyamov, M. Davletshin, R. Aipov, R. Jarullin, Journal of Mechanical Engineering Research and Developments 40(4), 706-715 (2007) DOI: 10.7508/jmerd.2017.04.018

12. E. Mednikov, Turbulent transport and deposition of aerosols (Science, Moscow, 1981) https://search.rsl.ru/ru/record/01001030187

13. A.M. Volk, Energetika, Proceedings of CIS higher educatiot institutions and power engineering associations 3, 77 (2009) https://energy.bntu.by/jour/article/view/499/493\# 